\title{
Solid Phase Synthesis of an Insect Pyrokinin Analog Incorpo- rating an Imidazoline Ring as Isosteric Replacement of a trans Peptide Bond
}

Krzysztof Kaczmarek ${ }^{1,2 *}$, Barbara Pacholczyk-Sienicka ${ }^{2}$, Łukasz Albrecht ${ }^{2}$, Janusz Zabrocki1 ${ }^{12}$, and Ronald J. Nachman ${ }^{1 *}$

1 Insect Control and Cotton Disease Research Unit, ARS, U.S. Department of Agriculture, 2881 F-B Road, College Station, TX 77845 USA;

2 Institute of Organic Chemistry, Lodz University of Technology, 90-924 Lodz, Poland;

* Correspondence: ron.nachman@usda.gov; krzysztof.kaczmarek@p.lodz.pl

Abstract: A facile solid-phase synthetic method for incorporating the imidazoline ring motif, a surrogate for a trans peptide bond, into bioactive peptides is reported. The example described is the synthesis of an imidazoline peptidomimetic analog of an insect pyrokinin neuropeptide via a cyclization reaction of an iminium salt generated from the preceding amino acid and 2,4-diaminopropanoic acid (Dap).

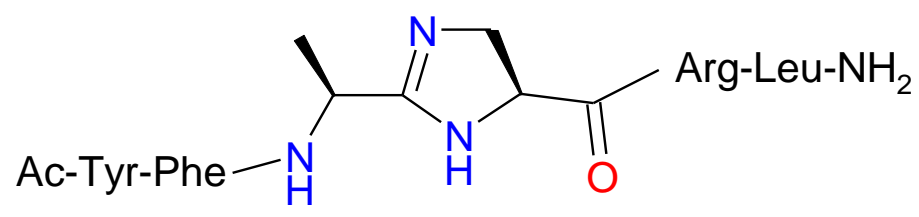

Keywords: insect neuropeptides; pyrokinins; trans peptide bond; imidazoline ring; SPOS;

\section{Introduction}

The imidazoline moiety has been previously introduced by Jones and coworkers as a peptide bond isostere (with an amidine as an amide bond replacement) [1,2]. Nachman et al. proposed that the imidazoline moiety can specifically function as a mimic or surrogate of the trans peptide bond, including a trans Pro, locking a trans orientation within the constrained five-membered imidazoline ring (Figure 1) [3-5].
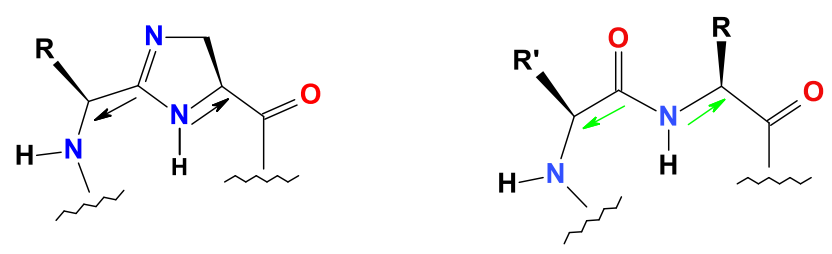

Figure 1. Comparison between trans-peptide bond structure (right) and imidazoline moiety (left) as a peptide bond replacement, and which mimics trans geometry irreversibly

However, whereas the molecular modeling suggests that the imidazole moiety can function as a mimic of a trans Pro, it is also clear that it is not an exact mimic [3-5]. Therefore, analogs containing the imidazoline moiety provide an opportunity for either a selective agonist interaction with closely related receptors of a superfamily or as an antagonist, as some receptors may display more tolerance to small deviations from the trans-peptide bond and/or trans Pro structure of natural peptides than others. 
The pyrokinin (PK) family of peptides plays a multifunctional role in the physiology of insects. In 1986 the first member of the family, leucopyrokinin (LPK), was isolated from the cockroach Leucophaea maderae [6] with over 30 members of this peptide class identified thereafter. All family members share the common $C$-terminal pentapeptide FXPRL-amide (X=S, T, G or V) and include subfamilies such as PKs, myotropins (MTs), PBAN, diapause hormone (DH), melanization and reddish coloration hormone (MRCH), pheromonotropin (PT), as well as pheromonotropic $\beta$ and $\gamma$ peptides derived from the cDNA of moths [7-10]. The PK family has been shown to stimulate sex pheromone biosynthesis in moths [7] and mediate critical functions associated with feeding (gut contractions) [10], development (egg diapause, pupal diapause and pupariation) [11-15] and defense (melanin biosynthesis) [16] in a variety of insects.

\section{Results and Discussion}

Incorporation of the imidazoline moiety into a PK/PBAN C-terminal hexapeptide sequence led to an analog, labeled PPK-Jo (Ac-YF[Jo]RLa)(Figure 2) [3,5]. PPK-Jo demonstrated strong activity in an in vivo S. littoralis melanotropic assay, matching the efficacy of PBAN and leucopyrokinin (LPK). Unlike the parent PK/PBAN hexapeptide YFTPRLa, PPK-Jo is a pure melanotropic agonist in the S. littoralis assay. PPK-Jo failed to elicit significant agonist (or antagonist) activity in three other related PK bioassays; i.e., an in vivo H. peltigera pheromonotropic assay, an in vivo N. bullata pupariation assay, and in an in vitro cockroach L. maderae hindgut myotropic assay $[3,5]$.

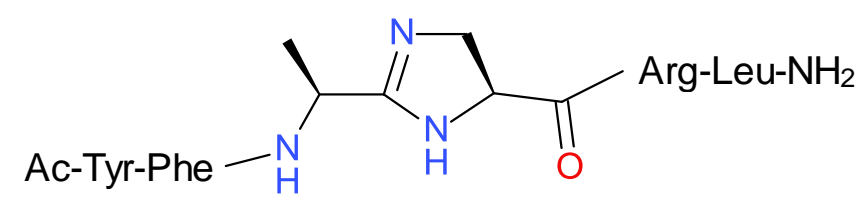

Figure 2. Structure of PPK-Jo, a selective agonist in one of four PK-related bioassays [3.5].

Another member of the PK neuropeptide family, the diapause hormone (DH), has been shown to terminate the protective state of diapause in pupae of heliothine moths [15]. The imidazoline motif was incorporated into the C-terminal heptapeptide active core region (LWFGPRLa) of DH [4,5], transforming a DH agonist into an antagonist capable of blocking the diapause termination activity of the native hormone in the corn earworm moth H. zea. The analog provides a lead for the development of DH analogs that can control insect pests via disruption of diapause behavior.

In this paper, we describe a solid phase synthetic (SPPS) route to incorporation of the imidazoline ring into the PK analog PPK-Jo (Ac-YF[Jo]RLa) (Figure 2). Solid phase synthesis on a polymer support offers advantages in that laborious purification at intermediate steps is eliminated and replaced by simple washing and filtration. In addition, the entire synthesis can be carried out in a single vessel, minimizing the loss of material during transfer from one vessel to another that would occur in solution phase synthesis. We decided to obtain the imidazoline motif through a cyclization reaction of an iminium salt generated from the preceding amino acid amide and 2,4-diaminopropanoic acid (1,3-diaminopropionic acid, Dap). Considering available solution phase syntheses leading to such rings that have been previously described $[1,2]$ which are very laborious, we decided to elaborate on the general procedure to allow the synthesis of such units in SPPS. Such a method would be very convenient for incorporation of the imidazoline unit into a wide range of peptide analogs.

\section{Materials and Methods}


We started with consecutive couplings of Fmoc-Leu-OH and Fmoc-Arg(Pbf)-OH to Fmoc-Rink-amide resin by means of HBTU/HOBt/DIEA as a reagent, in NMP as a solvent, using a $0.25 \mathrm{mmol}$ scale protocol (ABI) on an ABI 440 Peptide Synthesizer. The protocol for either manual or automated machines consists usually of the following, repeated operations for every single amino acid residue to be added to the growing peptide chain. These steps are: 1. deprotection of amino group of the amino acid, followed by a washing step; 2 . coupling (acylation) reaction of the next protected amino acid, followed by a washing step; 3. capping the unreacted, unacylated amino group, followed by a washing step.

After obtaining the dipeptide $\mathrm{H}-\mathrm{Arg}(\mathrm{Pbf})$-Leu- attached to Rink amide resin, we attempted to perform the coupling of Fmoc-Dap(Fmoc)-OH under the same conditions. Unfortunately, due presumably to the low solubility of the bis-Fmoc-1,3diaminopropanecarboxylic acid derivative in NMP, the coupling step performed on ABI synthesizer was not sufficiently efficient and this step had to be performed manually in a polypropylene syringe. After simultaneous removal of both fluorenylmethyleno-xycarbonyl (Fmoc) groups (20\% Piperidine/NMP, then washing with NMP (3x) and $\mathrm{MeOH}(3 \mathrm{x})$ and drying), both free amino groups were then allowed to react with a solution of iminium salt $\mathbf{2}$ derived from Fmoc-Ala- $\mathrm{NH}_{2} \mathbf{1}$ and triethyloxonium hexafluorophosphate or tetrafluoroborate (Figure 3). This reaction was also performed manually in the syringe. Fmoc-Ala- $\mathrm{NH}_{2} 1$ was synthesized from commercially available substrates and reagents, either from Fmoc-Ala-OSu by dissolving it in $\mathrm{MeOH}$ containing $1.1 \mathrm{eq}$ ammonia or from $\mathrm{H}-\mathrm{Ala}-\mathrm{NH}_{2}$ and Fmoc-OSu overnight in dioxane on a scale up to $20 \mathrm{mmol}$. After overnight stirring at rt followed by pouring into 5 volumes of water, product 1 precipitated quantitatively. The precipitate was collected by simple filtration and was extensively washed with water to remove the side product N-hydroxysuccinimide. Crude 1 was dried over phosphorus pentoxide in a desiccator to yield a white powder $\left(\mathrm{MH}^{+} 311\right.$, Kratos) with a purity over 99\% (HPLC) and was used for generation of the iminium salt as is.

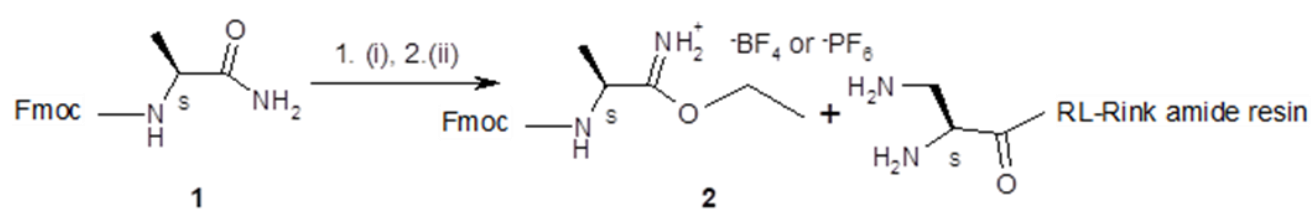

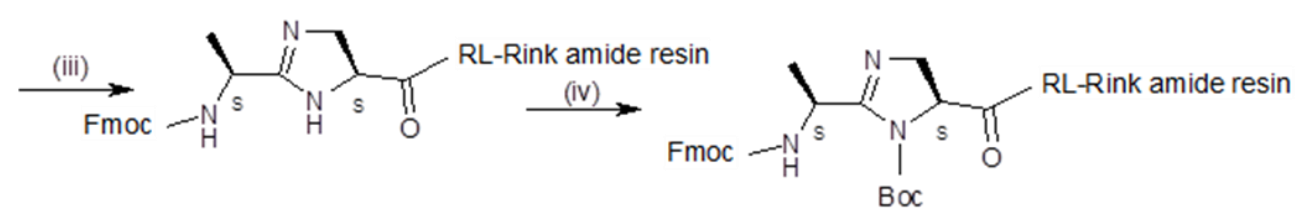

Figure 3. Synthesis of the iminoether 2 derived from Fmoc-L-Ala- $\mathrm{NH}_{2}$ 1, followed by ring formation on solid support: (i) 1.1 equiv. triethyloxonium tetrafluoroborate or triethyloxonium hexafluorophosphate in DCM, rt, 2h; (ii) washing with $1 \mathrm{M} \mathrm{KHCO}_{3}$ aq., overnight drying over anhydrous $\mathrm{MgSO}_{4}$; (iii) DIEA/DCM, overnight; (iv) Boc2 $\mathrm{O} / \mathrm{DIEA}, 3 \mathrm{~h}$.

Surprisingly, the imidazoline ring formation performed in DCM went well in contrast to NMP, which did not give the expected product at all. Reaction was carried out overnight manually Before removal of the Fmoc from the N-terminal nitrogen, the secondary nitrogen atom of the imidazoline ring was orthogonally protected to avoid an undesired acylation during the next coupling reaction. We checked the utility of two N-protecting groups - tert-Butyloxycarbonyl (Boc) and Trityl (Trt). They were introduced through a reaction with tert-butyl pyrocarbonate or trityl chloride in the presence of a tertiary amine, respectively. We found that during the cleavage of the pseudopeptide 
from the resin and final side chain deprotection, Boc protection gave products with higher yield and better purity.

Our synthetic route should not pose a larger racemization issue during SPPS than in other manual and automated SPPS syntheses, because we used HBTU as coupling reagent and $\mathrm{HOBt}$ as additive, which should suppress racemization. Moreover, the amino acids we used were protected with a urethane type group fluorenylmethylenoxycarbonyl (Fmoc). Our iminoether should be less prone to epimerization due to urethane-type protecting group. There is no reaction that involves the breaking of any bonds to a chiral center during cyclization to the imidazoline ring, and the alpha carbon atom of the Alanine residue is not a part of the imidazoline ring. Therefore, danger of epimerization of this chiral center should be diminished or even avoided. In the Supporting Information (SI), we have provided an HPLC chromatogram from the final purification step that shows a very small peak preceding the main product that features the same parent ion in the mass spectrum. We had surmised that this was likely an epimer and that this may indicate that the threat of racemization is minimal.

After the secondary imidazoline nitrogen was protected, the next standard steps for synthesizing the rest of the pseudopeptide sequence can be completed either manually in a syringe or on an automated peptide synthesizer. The cleavage of the final pseudopeptide from the resin with simultaneous deprotection of side chains was first attempted under standard conditions: a cocktail composed of TFA-TIS-Water (95/2.5/2.5), 2-4 h, rt, which gave no expected product, either due to reduction of the imidazoline double bond by silane or due to hydrolysis (presence of water in a cleavage cocktail). We found that a cocktail composed of TFA/DCM (1:1), 2-3 h, rt, used generally for removal of acid labile groups such as Boc, t-Bu, Trt in solution phase synthesis worked well for the cleavage of this pseudopeptide-resin complex. The final pseudopeptide product was purified on a Waters $\mathrm{C}_{18}$ Sep Pak cartridge and a Delta-Pak $\mathrm{C}_{18}$ reverse-phase columns. After lyophilization of combined fractions a white fluffy solid was obtained. The identity of the analog during the purification steps was confirmed via MALDI-MS on a Kratos Kompact Probe MALDI-MS machine (Kratos Analytical, Ltd., Manchester, UK) with the presence of the molecular ion $778.11\left[\mathrm{MH}^{+}\right]$. The structure was additionally confirmed after purification by high-resolution mass spectrometry (HRMS) and NMR (see Supporting Information). Calc. for $\mathrm{C}_{38} \mathrm{H}_{56} \mathrm{~N}_{11} \mathrm{O}_{7}=778.4264\left[\mathrm{MH}^{+}\right]$, found $778.4254\left[\mathrm{MH}^{+}\right]$ and $379.7226\left[\mathrm{MH}_{2}{ }^{2+}\right]$ for single and doubly charged ions, respectively. The presence of the imidazoline ring was confirmed based on 2D NMR spectroscopy. On the HMBC spectrum we observed a correlation peak between the methine proton $(\mathrm{CH}, 4.82 \mathrm{ppm})$ and the quaternary carbon atom of the imidazoline ring $(\mathrm{C}=\mathrm{N}, 146.50 \mathrm{ppm})$. Moreover, this carbon has also correlations with $\mathrm{CH}_{2}(3.72,4.10 \mathrm{ppm}), \mathrm{CH}_{3}(1.44 \mathrm{ppm})$ and $\mathrm{CH}(4.08$ ppm) groups (Figure 4).

Yield of the crude peptide attached to the polymer support, calculated from weight gain was approximately $90-95 \%$ in several trials on the scale from $0.05 \mathrm{mmol}$ to 0.125 mmol. Yield: $9-12 \%$ total of repurified product (HPLC) depending on the scale of synthesis. For the scale of 0.05 mmole the yield was $10 \%$ (5.12 micromoles). 
In conclusion, the described synthetic route provides a solid phase alternative to the introduction of an imidazoline ring motif as a trans-peptide bond surrogate into appropriate peptide sequences. It thus offers a useful tool to peptide chemists seeking to develop peptidomimetic analogs of biologically active peptides that can potentially feature selective and/or antagonist properties.

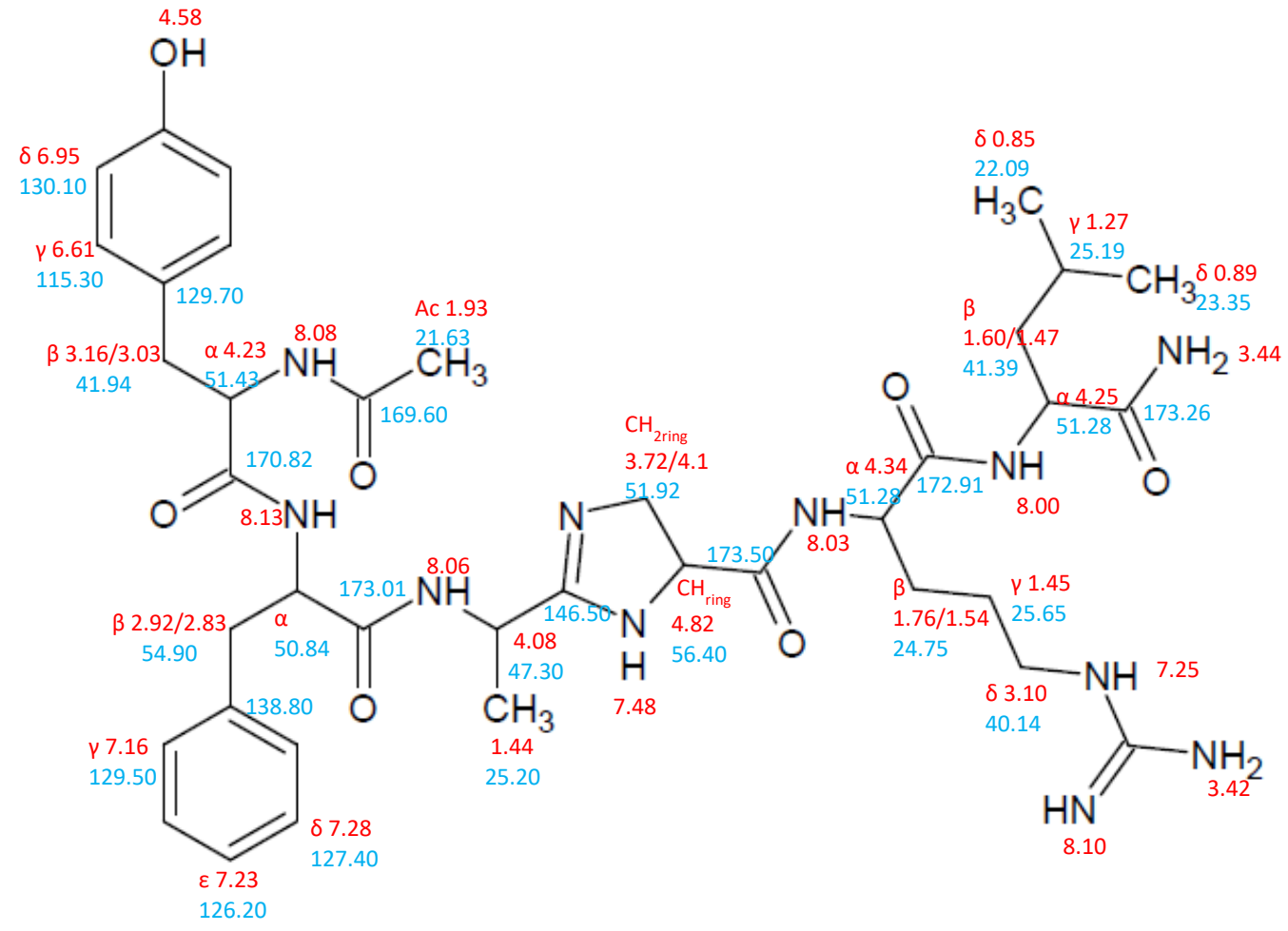

Figure 4. Structure of PPK-Jo with shifts of H (red) and C (blue) atoms displayed.

Supplementary Materials: The following are available online at www.mdpi.com/xxx/s1, SI KK RJN.pdf

Author Contributions: Conceptualization, K.K. and R.J.N.; methodology, K.K.; validation, K.K. and R.J.N.; formal analysis, K.K. and R.J.N; ;investigation, K.K., R.J.N.; resources, R.J.N.; data curation, B.P-S., R.J.N.; writing-original draft preparation, K.K., B.P.-S., R.J.N.; writing-review and editing K.K., J.Z., R.J.N.; visualization, K.K., B.P.-S..; project administration, R.J.N.; funding acquisition, L.A., R.J.N. All authors have read and agreed to the published version of the manuscript.

Funding: This research was funded by National Institute of Food and Agriculture (NIFA), United States Department of Agriculture grant number 2015-67013-23416 (RJN).

Acknowledgments: We thank Allison Strey (USDA) for technical assistance on peptide analog purification.

Conflicts of Interest: The authors declare no conflict of interest.

\section{References}

1. Jones, R. C. F.; Ward, G. J. Amide bond isosteres: Imidazolines in pseudopeptide chemistry. Tetrahedron Lett. 1988, 29(31), 3853-6. https://doi.org/10.1016/S0040-4039(00)82132-2

2. Gilbert,I. H.;Rees, D. C.; Crocket, A. K.; Jones, R. C. F. Imidazolines as amide bond replacements. Tetrahedron 1995, 51, 6315-36.

3. Nachman, R. J.; Ben-Aziz, O.; Davidovitch, M.; Kaczmarek, K.; Zabrocki, J.; Williams, H.; Strey, A. A.; Altstein, M. A novel dihydroimidazoline, trans-Pro mimetic analog is a selective PK/PBAN agonist. Frontiers In Bioscence. 2010, E2, $195-203$. DOI: $10.2741 / \mathrm{e} 82$

4. Zhang, Q.; Nachman, R. J.; Kaczmarek, K.; Zabrocki, J.; Denlinger, D. L. Disruption of insect diapause using agonists and an antagonist of diapause hormone. Proc. Natl. Acad. Sci. USA 2011, 108, 16922-6. DOI: 10.1073/pnas.1113863108 
5. Nachman, R. J. Peptidomics applied: A new strategy for development of selective antagonists/agonists of insect pyrokinin (FXPRLamide) family using a novel conformational-mimetic motif. EuPA Open Proteomics 2014, 3, 138-142. https://doi.org/10.1016/j.euprot.2014.02.008

6. Holman, G. M.; Cook, B. J.; Nachman, R. J. Primary structure and synthesis of a blocked myotropic neuropeptide isolated from the cockroach, Leucophaea maderae. Comparative Biochemistry and Physiology Part C: Comparative Pharmacology. 1986, 85(1), $219-224$. https://doi.org/10.1016/0742-8413(86)90077-0

7. Altstein, M. Role of neuropeptides in sex pheromone production in moths. Peptides 2004, 25, 1491-1501. DOI: $\underline{10.1016 / j . p e p t i d e s .2004 .06 .020}$

8. Gäde, G.; Hoffmann, K. H.; Spring, J. H. Hormonal regulation in insects: facts, gaps, and future directions. Physiol. Rev. 1997, 77, 963-1032. DOI: $10.1152 /$ physrev.1997.77.4.963

9. Predel, R.; Russell, W. K.; Neupert, S.; Russell, D. H., Esquivel, J. F. and Nachman, R. J. Identification of the first neuropeptides from the CNS of Hemiptera: CAPA peptides of the southern green stinkbug Nezara viridula (L.). Peptides 2006, 27, 2670-7. DOI: $10.1016 /$ j.peptides.2006.05.013

10. Paluzzi, J.; Park, Y.; Nachman, R. J.; Orchard, I. Isolation, expression analysis, and functional characterization of the first antidiuretic hormone receptor in insects. Proc. Natl. Acad. Sci. USA 2010, 107, 10290-5. DOI: 10.1073/pnas.1003666107

11. Nachman, R. J.; Holman, G. M.; and Cook, B. J. Active fragments and analogs of the insect neuropeptide leucopyrokinin: structure-function studies. Biochem. Biophys. Res. Commun. 1986, 137, 936-42. DOI: 10.1016/0006-291x(86)90315-3

12. Imai, K.; Konno, T.; Nakazawa Y.; Komiya, T.; Isobe, M.; Koga, K,; Goto, T.; Yaginuma, T.; Sakakibara, K.; Hasegawa, K.; Yamashita, O. Isolation and Structure of Diapause Hormone of the Silkworm, Bombyx mori. Proc. Jpn. Acad., Ser. B 1991, 67, 98-101. https://doi.org/10.2183/pjab.67.98

13. Nachman, R. J.; Holman, G. M.; Schoofs, L.; Yamashita, O. Silkworm diapause induction activity of myotropic pyrokinin (FXPRLamide) insect neuropeptides. Peptides 1993, 14, 1043-8. DOI: 10.1016/0196-9781(93)90084-t

14. Nachman, R. J.; Zdarek, J.; Hayes, T. K.; Holman, G. M. Pupariation Acceleration in Fleshfly (Sarcophaga bullata) Larvae by the Pyrokinin/PBAN Neuropeptide Family. Ann. N.Y. Acad. Sci. 2006, 814, 73-79. DOI: 10.1111/j.1749-6632.1997.tb46146.x

15. Xu, W. H.; Denlinger, D. L. Molecular characterization of prothoracicotropic hormone and diapause hormone in Heliothis virescens during diapause, and a new role for diapause hormone. Insect Mol. Biol. 2003, 12, 509-16. DOI: $\underline{10.1046 / j .1365-2583.2003 .00437 . x}$

16. Altstein, M.; Gazit, Y.; Ben Aziz, O.; Gabay, T.; Marcus R.; Vogel Z.; Berg, J. Induction of cuticular melanization in Spodoptera littoralis larvae by PBAN/MRCH: Development of a quantitative bioassay and structure function analysis. Arch. Insect Biochem. Physiol. 1996, 31, 355-70. https://doi.org/10.1002/(SICI)1520-6327(1996)31:4<355::AID-ARCH1>3.0.CO;2-V 